\title{
Flipping the classroom: Case-based learning, accountability, assessment, and feedback leads to a favorable change in culture
}

Nahush A. Mokadam, MD, ${ }^{a}$ Todd F. Dardas, MD, MS, ${ }^{b}$ Joshua L. Hermsen, MD, ${ }^{a}$ Jay D. Pal, MD, PhD, ${ }^{a}$ Michael S. Mulligan, MD, ${ }^{\mathrm{a}}$ L. Myria Jacobs, MLIS, ${ }^{\mathrm{a}}$ Douglas E. Wood, MD, ${ }^{\mathrm{a}}$ and Edward D. Verrier, $\mathrm{MD}^{\mathrm{a}}$

\section{ABSTRACT}

Objective: The 88-week Thoracic Surgery Curriculum is challenging to implement because of the large content in a traditional lecture format. This study investigates flipping the classroom by using a case-based format designed to stimulate resident preparation and engagement.

Methods: The didactic conference format was altered. Curricular reading assignments, case review, and conference participation prepared residents for novel formative assessment quizzes. Ten residents participated, and faculty served as controls. Scores were compared with the use of linear regression adjusted for clustering of responses for each person. A survey was administered to determine impressions of this educational technique.

Results: A majority of residents completed curricular readings (82\%) and reviewed case presentations $(79 \%)$. Resident performance initially lagged behind faculty but exceeded faculty performance by the conclusion (interaction $P=.047)$. Junior resident overall performance was superior to senior residents over the entire analysis $(P=.026)$; however, both groups improved over time similarly $(P=.34)$ Increased reading from the curriculum $(5 \%$ increase per level, $P=.001)$ and case presentation review $(6 \%$ increase per level, $P<.0001)$ were associated with improved quiz performance. Residents presenting cases at their session performed no better than other quiz-takers for the same session $(P=.38)$. The majority of residents viewed this method favorably.

Conclusions: This method stimulated increased resident participation and engagement in this pilot study. Assessment scores increased at both resident levels, and resident performance exceeded faculty performance with time. By using experiential learning principles, flipping the classroom in this manner may improve educational culture by enhancing accountability, assessment, and feedback. (J Thorac Cardiovasc Surg 2017;153:987-96)

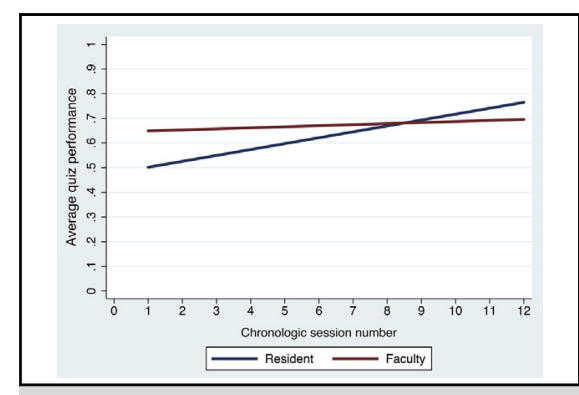

Improving resident performance over time compared with faculty.

\section{Central Message}

By the use of experiential learning principles, flipping the classroom may improve educational culture by enhancing accountability, assessment, and feedback.

\section{Perspective}

In a pilot study, using a case-based format of curricular teaching, residents prepared and reviewed material while increasing their engagement and scores on quizzes and ultimately exceeded faculty performance. By the use of experiential learning principles, flipping the classroom may improve educational culture by enhancing accountability, assessment, and feedback.

See Editorial Commentary page 997
The curricular requirements set forth by the American Board of Thoracic Surgery (ABTS) for the completion of suitable cardiothoracic training have been revised recently. ${ }^{1}$ This new paradigm was the basis for the redevelopment of

\footnotetext{
From the Divisions of ${ }^{\mathrm{a}}$ Cardiothoracic Surgery and ${ }^{\mathrm{b}}$ Cardiology, University of Washington, Seattle, Wash.

Read at the 42nd Annual Meeting of the Western Thoracic Surgical Association, Waikoloa, Hawaii, June 22-25, 2016.

Received for publication May 26, 2016; revisions received Oct 10, 2016; accepted for publication Oct 17, 2016; available ahead of print Jan 11, 2017.

Address for reprints: Nahush A. Mokadam, MD, Division of Cardiothoracic Surgery, University of Washington, Box 356310, Suite AA115 1959, NE Pacific St, Seattle, WA 98195 (E-mail: mokadamn@uw.edu). $0022-5223 / \$ 36.00$

Copyright (C) 2016 by The American Association for Thoracic Surgery http://dx.doi.org/10.1016/j.jtcvs.2016.10.101
}

the so-called 88-week curriculum by the Thoracic Surgery Directors Association and incorporation of this curriculum by the Joint Council for Thoracic Surgery Education (the Joint Council for Thoracic Surgery Education was incorporated into the Society of Thoracic Surgeons on July 1, 2016) into their new learning and content management systems and the subsequent thoracic surgery curriculum (TSC). ${ }^{2,3}$ This curriculum has oversight from an editorial board and

Scanning this $\mathrm{QR}$ code will take you to the videos and appendix for the article.

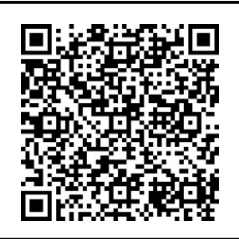




\section{Abbreviations and Acronyms \\ ABTS $=$ American Board of Thoracic Surgery \\ $\mathrm{CI}=$ confidence interval \\ LMS = learning management system \\ $\mathrm{TSC}=$ thoracic surgery curriculum}

consists of book chapters, peer-reviewed journal articles, lectures, and reviews.

It has been recognized by educators and learners alike that the current curricular content has the potential to be overwhelming in volume and scope. As an example, the content for "Management of Benign Esophageal Disorders III," includes 3 book chapters, 2 review articles, and 2 peerreviewed publications. ${ }^{4}$ Because many programs focus their teaching conferences based on progress in the curriculum, content covered during these sessions can devolve into dense lectures driven by a perceived need to cover all the assigned content. ${ }^{5}$ At our institution, we have used didactic (traditional lectures), case-based presentations, review of standardized examinations, and other educational modalities to maintain resident and faculty engagement. It had been noted by our faculty and residents that the casebased presentations often were the most dynamic and interactive. Partially driven by this observation, more than 100 case-based presentations were incorporated into the TSC to help guide teaching sessions. ${ }^{4}$ Anecdotally, these cases were used sparingly, and there was little resident accountability by the TSC platform for the entire content matter. ${ }^{6}$ Furthermore, the cases were criticized as lacking assessment and feedback capabilities. Although the original learning management system (LMS) for the TSC did have quiz functions, it lacked robust reporting tools thereby limiting its overall value. This year, a new LMS was launched with additional functionality that has yet to be explored fully.

It also has been recognized in our specialty (and others) that traditional methods of teaching have faced significant challenges over the last decade. ${ }^{5}$ Flipping the classroom is characterized by learners engaged in active learning and assessment with feedback and increasingly has been used in many environments. ${ }^{7}$ It requires the learners to acquire the majority of content during nonclass time, so that direct teacher-student interactions are optimized.

This pilot study sought to examine a method by which to engage residents and faculty in educational conferences that required case-based learning via a new conference format, incorporated elements of assessment by including quizzes, promoted accountability for content by querying resident preparation and self-assessment, and provided meaningful feedback by providing live feedback during conferences and by furnishing quiz results. After a thorough review of the literature, we believed this teaching style would be well suited to our cardiothoracic educators and trainees, given the limited work hours and breadth and depth of content. We elected to pursue this strategy because of our existing experiences with case-based learning, its intuitive nature to our (local) teaching style, and the incorporation of cases embedded in the TSC, as this could provide the basis for other educators on their use.

\section{METHODS}

\section{Conference Conduct}

In a prospective fashion, over the course of 12 educational sessions ( 5 cardiac, 7 thoracic), the regularly scheduled didactic conference format was altered. A resident-faculty dyad was created for each conference. Together, they prepared an illustrative case covering the assigned topic, some of which were derived directly from the TSC. (Example presentation from "Management of Benign Esophageal Disorders III": Video 1$)^{8}$ Reading assignments, based on the curriculum, and focused on the case, as well as the case itself, were distributed to the residents (6 integrated, 4 traditional, or 4/3) and faculty for preview and preparation before the conference date. Via an interactive format, these topics were dissected by all members at the educational session as follows: After learning objectives are outlined, a case presentation is performed with pertinent findings. Both basic and advanced elements of the history, physical, and workup are included. The Socratic method is used by faculty to gauge level of understanding in learners of all levels. Diagnostic and therapeutic maneuvers are discussed in a group format, including risks and benefits of surgical options. Residents of all levels are given opportunities to ask questions and solicit faculty opinions in clinically vague areas. The case is then summarized and learning points once again highlighted.

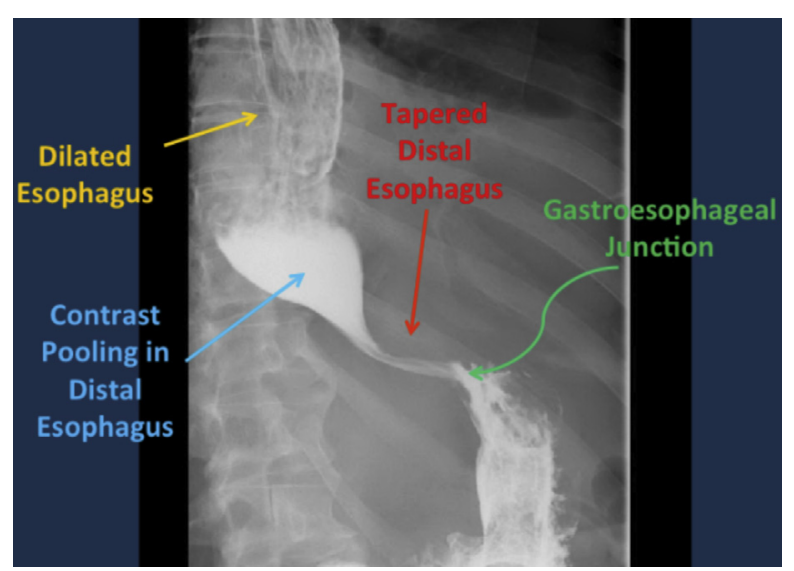

VIDEO 1. A video of the slide set for from "Management of Benign Esophageal Disorders II" conference is included. After learning objectives are outlined, background reading is specifically identified from within the TSC. A case presentation is performed with pertinent findings. Both basic and advanced elements of the history, physical, and workup are included so as to engage learners of multiple levels. An in-depth discussion of manometry is conducted, which then leads to a discussion. The discussion includes therapeutic options and strategies, as well as surgical options. The case is summarized and learning points once again highlighted. Video available at: http://www.jtcvsonline.org/article/S0022-5223(16)31681-6/addons. 


\section{Quiz Preparation}

A multiple-choice quiz was developed by the assigned faculty member to assess knowledge of the curriculum (Example quiz from "Management of Benign Esophageal Disorders III": Video 2). The quiz items were newly written by the faculty to cover the assigned topic and were not obtained from commercially available sources. In all instances, the quiz content went beyond the content from the conference alone and was inclusive of the readings from the curriculum. Quizzes were distributed at the end of conference to all the residents (whether or not they were present at conference) and, as a control, to nonpresenting faculty members of the respective subspecialty - cardiac surgery faculty only took cardiac surgery quizzes, and thoracic surgery faculty only took thoracic surgery quizzes. A brief 4-question survey accompanied the quiz to quantify resident readings from the curriculum, case review before completing the quiz, conference attendance, and self-assessment of competence (using the modified Dreyfus scale) in the topic. Residents and faculty received a report of their individual performance on the quizzes.

\section{Survey}

A survey consisting of 22 questions was administered anonymously to the residents, which explored demographics, career interests, conference preferences, preparation, and was specifically designed to evaluate the impact of the change in conference conduct (Appendix E1).

\section{Analysis}

Each resident's pre- and post-quiz engagement, self-assessment, and attendance were recorded for each quiz. Simple linear regression was used to evaluate engagement with preparation and review materials, selfassessment, and attendance over time. Analysis of accuracy was performed by the use of fixed-effects linear regression to adjust for clustering of responses at the level of residents or faculty. Interactions between successive sessions and seniority were performed based on visual inspection graphs of performance over time by seniority, which suggested differential accuracy by seniority and faculty/resident status. Predicted test scores were derived from the linear models to demonstrate the effect of engagement, attendance, and self-assessment. Stata (version 13; Stata Corp, College Station, Tex) was used for all analyses.

\section{Institutional Review Board}

The University of Washington institutional review board granted a waiver for these activities.

\section{RESULTS}

Residents were well distributed across years of training, with 4 residents in their final 2 years of training (senior residents) and 6 residents with more than 2 years remaining

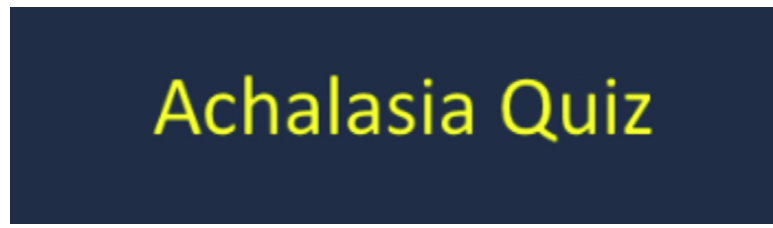

VIDEO 2. Corresponding quiz items from "Management of Benign Esophageal Disorders II" conference. The quiz includes multiple choice questions and true/false questions. These items are designed to draw from the presentation and the reading materials for the session. Video available at: http://www.jtcvsonline.org/article/S0022-5223(16)31681-6/ addons. (junior residents). Of the residents, $70 \%$ were male, and career interests were $30 \%$ adult cardiac, $20 \%$ general thoracic, $10 \%$ congenital, $10 \%$ cardiothoracic, and $30 \%$ undecided. One-half of the residents anticipated an academic career, whereas the remaining were undecided.

Quiz performance improved with time as residents gained experience with the system (Figure 1). Resident performance was not statistically different from faculty over the course of the program (coefficient for faculty 0.057 , $95 \%$ confidence interval $[95 \% \mathrm{CI}]-0.03,0.15, P=.22$ ); however, resident performance was lower than faculty initially but exceeded faculty performance by the end of the program (faculty*session interaction $P=.047$, Figure 2, $A$ ). Junior resident overall performance was superior to senior residents over the entire analysis (seniority coefficient $-0.1 ; 95 \% \mathrm{CI},-0.19,-0.01 ; P=.026$ ); however, both groups improved over time at a similar rate (seniority* session interaction $P=.34$, Figure 2, $B$ ).

A majority of residents studied the readings from the curriculum before conference $(82 \%)$ and reviewed the case presentations after conference $(79 \%)$ before their assessment, whereas more than one-third of residents were absent from conference $(35.7 \%)$. The majority of residents $(66.9 \%)$ considered themselves novice or advanced beginners (Table 1). Increased reading from the curriculum (coefficient $0.05 ; 95 \% \mathrm{CI}, 0.02-0.08, P=.001)$ and increased case presentation review (coefficient $0.06 ; 95 \%$ CI, 0.03-0.08, $P<.0001)$ were associated with improved quiz performance. Residents presenting cases demonstrated a nonsignificant signal toward improvement on quizzes compared with other quiz-takers for the same session (vs absent coefficient $0.1, P=.38$, vs present coefficient

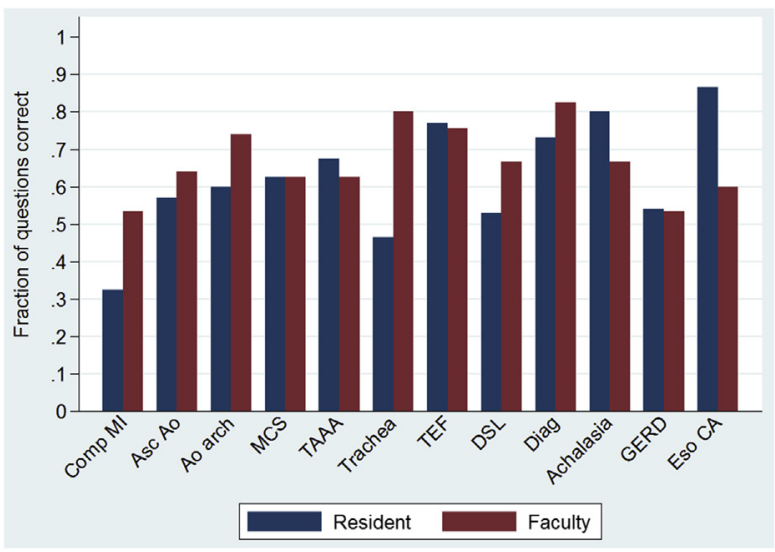

FIGURE 1. Comparison of scores between residents and faculty. Comp $M I$, Complications of myocardial infarction; Asc Ao, ascending aortic disease; Ao arch, aortic arch; MCS, mechanical circulatory support; TAAA, thoracoabdominal aortic aneurysm; Trachea, tracheal disease; TEF, tracheoesophageal fistula; $D S L$, diverticulae-stricture-lye; Diag, esophageal anatomy; diagnostic tools; GERD, gastroesophageal reflux disease; Eso CA, esophageal cancer. 


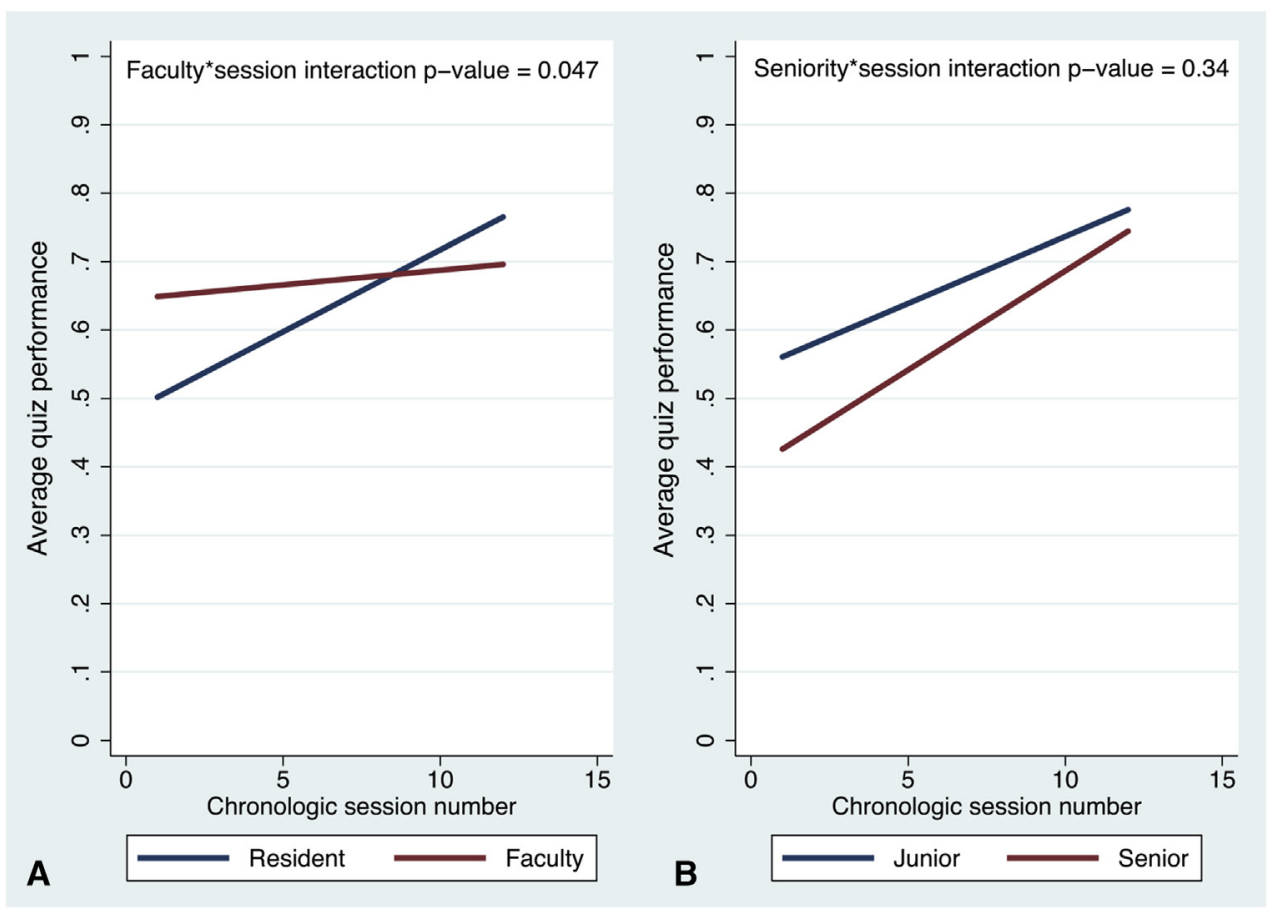

FIGURE 2. Comparison of resident and faculty quiz score trends over time. Lines are derived from simple linear regression, whereas the interaction $P$ values (faculty*session and seniority*session) were estimated from a fixed-effects model. A, Comparison of residents to faculty over time. B, Comparison of junior with senior residents over time. Junior residents overall performed better than senior residents $(P=.026)$.

$0.05, P=.68)$. Power to detect differences was limited to analysis of only 12 sessions. Resident self-assessment of increasing proficiency with material was consistent with performance on quizzes (coefficient 0.05 ; $95 \%$ CI, 0.01$0.09, P=.018$ ). Predicted test performance scores for each level of engagement, attendance, and self-assessment also are provided. For the entire group, average engagement with case readings $(P=.84)$, case presentation reviews $(P=.954)$, and self-assessment values $(P=.129)$ did not change over the course of the program.

Seventy percent of the residents viewed this conference format as superior to formal resident or faculty didactic sessions, review of standardized questions, or guest lectures. The majority of the residents $(60 \%)$ reported that the case-based format motivated them to prepare for conference, despite the fact that $60 \%$ also believed that the TSC reading assignments were "too much." Multiple residents cited faculty engagement as one of the most beneficial aspects of this effort. Multiple residents also sought more elaborate feedback than was provided. Almost all the residents commented that they were motivated by this process to prepare more overall.

\section{DISCUSSION}

The overarching aim of this study is to move the needle in changing the culture of didactic education in cardiothoracic surgery. Surgical culture has been studied qualitatively and quantitatively in other domains, generally related to operating room performance and changes in clinical outcomes, with encouraging, but mixed, results ${ }^{9,10}$; however, much of the attention in our specialty has focused on culture at a national level-duty hours, ABTS pass rates, and innovative ways for acquisition of surgical skills. ${ }^{11-13}$ The TSC provides a framework for national culture change, but this does not equate to direct transmission of that change to implementing programs. For a curriculum to be complete, it requires a needs assessment, objectives, assessment of the learner, and assessment of the curriculum itself. ${ }^{14}$ This pilot study allowed our institution the opportunity to evaluate the TSC (and more specifically, case-based education) in a manner not previously done.

Substantial attention decline occurs in as little as $10 \mathrm{mi}-$ nutes, and even in high-functioning medical students, only lasts an average of 20 minutes. ${ }^{15,16}$ Furthermore, lecturebased information has a low overall retention rate of about $20 \%$, making the traditional hour-long lecture an inefficient mechanism by which to teach complex topics such as cardiothoracic surgery. ${ }^{17}$ Experiential learning has emerged as one important element of education, which draws on understanding and rationale, rather than traditional dictum. This is in contrast to the concept of knowledge transmission during didactic learning, which is the basis for our traditional education, and referred to by our faculty as the "dump truck of knowledge." Experiential learning theory 
TABLE 1. Resident preparation, attendance, and self-assessment for quizzes and effect on quiz scores

\begin{tabular}{lcccc}
\hline & Percent & $\begin{array}{c}\text { Regression } \\
\text { coefficient }\end{array}$ & $\begin{array}{c}\text { Predicted } \\
\text { quiz score }\end{array}$ & $\boldsymbol{P}$ value \\
\hline Readings from curriculum & & & & \\
Read all & 9.8 & 0.05 & $72.6 \%$ & .001 \\
Most & 26.8 & & $67.6 \%$ & \\
Some & 34.8 & & $62.6 \%$ & \\
Little & 10.7 & & $57.6 \%$ & \\
None & 17.9 & & $52.6 \%$ & \\
Case review before quiz & & & & \\
All reviewed & 33 & 0.06 & $72.1 \%$ & $<.0001$ \\
Most & 19.6 & & $66.4 \%$ & \\
Some & 18.8 & & $60.7 \%$ & \\
Little & 8 & & $55.0 \%$ & \\
None & 20.5 & & $49.3 \%$ & \\
Attendance at conference & \multicolumn{3}{c}{} & \\
Presented & 2.7 & Reference & $71.9 \%$ & \\
Attended & 54.5 & -0.05 & $67.2 \%$ & .684 \\
Absent & 35.7 & -0.10 & $61.6 \%$ & .379 \\
Self-assessment of competence on topic & & \\
Proficient & 10.7 & 0.05 & $72.2 \%$ & .018 \\
Competent & 22.3 & & $67.2 \%$ & \\
Advanced beginner & 33.9 & & $62.2 \%$ & \\
Novice & 33 & & $57.2 \%$ & \\
\hline
\end{tabular}

Regression coefficients correspond to improvement in performance (quiz percentage score) by each activity or versus all other groups in the case of attendance. Predicted test performance scores were based upon resident reported preparation, participation, and confidence.

is based on the following concepts: learning is a process that requires engagement and feedback; all learning is relearning; learning requires resolution of conceptual conflict; learning involves integrative functioning; learning results from synergy between the learner and the environment; learning is the process of creating knowledge. ${ }^{18}$ The concepts of experiential learning theory are integral in adult learning theory-active involvement in goals, building on previous knowledge, intrinsic motivation, and responsibility. ${ }^{19}$

The benefit of learner engagement by altering the traditional lecture-based teaching style has been studied in other settings. In a physics class, the use of preclass reading, questions, discussions, and feedback increased student class attendance and increased scores compared with control subjects. ${ }^{20}$ This also has been observed in meta-analysis examining the effectiveness of Continuing Medical Education. ${ }^{21}$ In that study, the use of didactic sessions did not translate into changes in performance or differences in healthcare outcomes. This finding is in contrast to the observation that case discussions and hands-on sessions were more effective and led to a change in behavior and practice. It is also notable that experiential learning is one type of learning that may be complementary other learning types, or may be more or less effective in certain individuals depending on their individual learning styles.
Interestingly, junior residents performed better overall than senior residents in this study. This may be attributed to time constraints in the more clinically demanding senior years, junior resident enthusiasm and thirst for cardiothoracic content, or other factors. It is encouraging that naïve learners in cardiothoracic surgery can be educated with regard to complex concepts when using novel educational methods. Equally interesting is the observation that resident performance, through preparation, exceeded faculty performance on some of these assessments. From the perspective of educators, this may be the most encouraging finding in the study-if the trainee can achieve the competence of the educator, then for this point in time, the trainee has learned and advanced towards competence. This also could indicate acquisition of expertise in this process (test-taking skills) without necessarily reflecting expertise in content over the short or long term. Indeed, it may be surprising that 3 groups (faculty, senior residents, presenting residents) who would have a priori been predicted to perform better on exams in fact did not. This may reflect limitations in the assessment, or may also reflect a higher level of understanding that exceeded the fidelity of a series of multiple-choice questions.

This study incorporated these experiential learning principles into the cardiothoracic training arena to enact culture change. This process stimulated increased resident and faculty engagement. This increased engagement may be responsible for the overall satisfaction results seen in the survey. Nonetheless, over time, both junior and senior residents improved performance at an equivalent rate, suggesting synergy between the learner and the environment. This synergy also may be a product of a relatively large resident cohort incorporating learners of many levels. Extrapolation of these data to other programs bears further study.

Not surprisingly, but statistically meaningful, reading from the curriculum, reviewing the cases (relearning), attending conference (resolution of conceptual conflict), and overall confidence (creating knowledge) predicted greater scores (feedback) in residents of all levels. The majority of residents had a favorable impression of this process and reported that it motivated them both to prepare before and review after the weekly teaching conferences. The new TSC LMS may enable this type of assessment and feedback with its ability to have benchmark quizzes and normalize the results across the national sample. These data also may provide evidence to encourage educators of any level (including those who have "always done it this way") to consider other teaching styles in their curricula. Furthermore, use of the case-based presentations already embedded in the TSC can provide an ample starting point from which to begin. All culture is of course local culture, and the experience described in this paper may best serve as a blueprint for recapitulation in the broader surgical 
educational arena. It may be reasonable to examine the goals, objectives, and motivators of the educators to better define teaching practices in future studies. There also may be an opportunity to further explore this methodology in a multicenter fashion.

\section{Limitations}

Although this study was prospective, it was not possible to provide resident-level control observations. Furthermore, the assessment quizzes used were not subject to psychometric evaluation and therefore cannot be extrapolated to performance on standardized examinations such as the Thoracic Surgery Directors Association in-training examination or the ABTS qualifying examination. There was no analysis of long-term knowledge retention that may benefit from independent investigation. Finally, this is a preliminary pilot evaluation of this methodology for our specialty and warrants further study.

\section{CONCLUSIONS}

Using a case-based format of curricular teaching, residents prepared and reviewed material while increasing their engagement and scores on quizzes and ultimately exceeded faculty performance. By using experiential learning principles, flipping the classroom may improve educational culture by enhancing accountability, assessment, and feedback.

\section{Conflicts of Interest Statement}

Dr Mokadam is an investigator for St Jude Medical, HeartWare, and Syncardia and a consultant for St Jude Medical and HeartWare. Dr Dardas has research support from ISHLT/HeartWare. Dr Pal is a consultant for HeartWare. All other authors have nothing to disclose with regard to commercial support.

\section{References}

1. American Board of Thoracic Surgery. Curriculum. Available at: https://www. abts.org/root/home/curriculum.aspx. Accessed April 16, 2016.

2. Thoracic Surgery Directors Association. Thoracic surgical curriculum. Available at: http://www.tsda.org/education/thoracic-surgery-curricula. Accessed April $16,2016$.

3. Joint Council on Thoracic Surgery Education. Thoracic surgical curriculum. Available at: http://www.jctse.org/education/thoracic-surgical-curriculum. Accessed April 16, 2016.

4. Joint Council on Thoracic Surgery Education. JCTSE online course. Available at: http://jctse.mrooms.net. Accessed April 16, 2016.

5. Vaporciyan AA, Yang SC, Baker CJ, Fann JI, Verrier ED. Cardiothoracic surgery residency training: past, present and future. J Thor Cardiovasc Surg. 2013;146: 759-67.

6. Antonoff MB, Verrier ED, Allen MS, Aloia L, Baker C, Fann JI, et al. Impact of Moodle-based online curriculum on thoracic surgery in-training examination scores. Ann Thor Surg. 2016;102:1381-6.

7. McLaughlin JE, Roth MT, Glatt DM, Gharkholonarehe N, Davidson CA, Griffin LM, et al. The flipped classroom: a course redesign to foster learning and engagement in a health professions school. Acad Med. 2014;89:236-43.

8. Yates R, Verrier E. Achalasia. In: Thoracic Surgery Curriculum, TS 34: Management of Benign Esophageal Disorders III. Available at: http://learnctsurgery.sts. org/lms/libraries. Accessed August 25, 2016.
9. Bosk C. Forgive and Remember: Managing Medical Failure. Chicago: University of Chicago Press; 1979: 2003.

10. Yule S, Sacks GD, Maggard-Gibbons M. Innovative approaches for modifying surgical culture. JAMA Surg. 2016;151:791-2.

11. Connors RC, Doty JR, Bull DA, May HT, Fullerton DA, Robbins RC. Effect of work-hour restriction on operative experience in cardiothoracic surgical residency training. J Thorac Cardiovasc Surg. 2009;137:710-3.

12. Moffatt-Bruce SD, Ross P, Williams TE. American Board of Thoracic Surgery examination: fewer graduates, more failures. J Thorac Cardiovasc Surg. 2014; 147:1464-70.

13. Mokadam NA, Lee R, Vaporciyan AA, Walker JD, Cerfolio RJ, Hermsen JL, et al. Gamification in thoracic surgical education: using competition to fuel performance. J Thorac Cardiovasc Surg. 2015;150:1052-8.

14. Vaporciyan AA. Teaching and learning surgical skill. Ann Thor Surg. 2016;101: $12-4$.

15. Stuart J, Rutherford RJ. Medical student concentration during lectures. Lancet. 1978:312:514-6.

16. Hartley J, Cameron A. Some observations on the efficiency of learning. Educ Rev. 1967;20:30-7.

17. Hartley J, Davies JK. Note-taking: a critical review. Innov Educ Train Int. 1978; 15:207-24.

18. Kolb AY, Kolb DA. Learning styles and learning spaces: enhancing experiential learning in higher education. Acad Manag Learn Educat. 2005;4:193-212.

19. Goldman S. The educational Kanban: promoting effective self-directed adult learning in medical education. Acad Med. 2009;84:927-34.

20. Deslauriers L, Schelew E, Wieman C. Improved learning in a large-enrollment physics class. Science. 2011;332:862-4.

21. Davis D, O'Brien MAT, Freemantle N, Wolf FM, Mazmanian P, Taylor-Vaisey A. Impact of formal continuing medical education: do conferences, workshops, rounds, and other traditional continuing education activities change physician behavior or health care outcomes? J Am Med Assoc. 1999;282:867-74.

Key Words: curriculum, motivation, pilot projects, problem-based learning, thoracic surgery

\section{Discussion}

WTSA Day 2 1st Break to End of Day

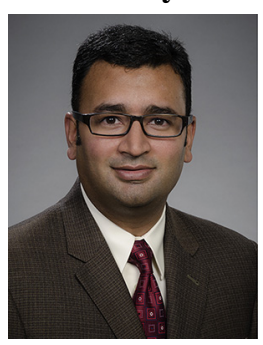

Dr Nahush A. Mokadam

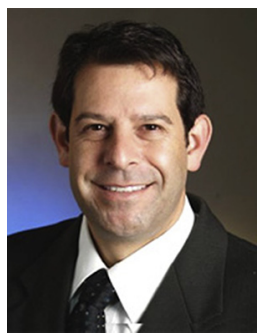

Discussant: Dr Craig Baker

Discussant 2: Dr David Fullerton

Discussant 3: Dr Richard Whyte

Moderator: Dr Jessica S. Donington

Presenter: Dr Nahush A. Mokadam

Moderator: Our next paper is, "Flipping the Classroom, Case Based Learning Accountability Assessment and 
Feedback," presented by Dr Mokadam from his group from Seattle.

Moderator: Dr T. Brett Reece. Just to warn you, Marv Pomerantz threatened me to keep everybody on time so we will.

Dr Mokadam. I'd like to thank the Association for the opportunity to present our work. We have no relevant financial disclosures, but all of the authors are invested heavily in education and many of us were involved intimately in the development of the thoracic surgery curriculum. The thoracic surgery curriculum was derived from the original blue book and is an 88week curriculum from the Thoracic Surgery Directors Association. This has been assumed by the Joint Council on Thoracic Surgery Education and has been managed by an editorial board over the last several years. It's housed in a Moodle learning management system and is sourced from a content management system called the "brain." It has been recognized that this curriculum currently has an overwhelming volume of content. It also does not have robust feedback mechanisms to give the learner or the program directors. This, coupled with work hour restrictions and disengagement of learners and faculty due to all of our distractions, has raised alarms with the decreasing ABTS pass rate.

This is a screen shot of 1 week or one session from the thoracic surgery curriculum. As you can see, it includes several book chapters, several publications, and lectures. This is coupled by death by PowerPoint at 6:30 in the morning on Friday at the end of a long week. You have curriculomegaly, sometimes uninspired presenters, which often results in sleeping in the classroom, and lack of engagement of the learners. Well, a study was conducted in a large physics class, as an example, and they took about 300 students in each class and they divided them. They put one group in a lecturebased format with the highest-rated evaluator, the most honored lecturer giving all the talks, and the other group was led by the teaching assistants, giving preclass quizzes, in class question and answer discussions, and targeted instructor feedback. When they compared the 2 groups on an examination, you can see that the traditional lecture-based model underperformed the teaching assistant-based interactive model.

Recognizing this, the TSC also has incorporated into it almost 200 cases looking at various different aspects of cardiothoracic surgery and this is just another example of some of the lectures that are available. For those of you that are not familiar with this, these actually exist as PowerPoint slides that can be downloaded freely and modified for anybody to use. Experiential adult learning theory is based on these principles. It requires engagement and feedback, repetition, resolution of conceptual conflict such as discussion, integrating concepts, getting synergy between the learner, the environment, and the teacher, which ultimately creates knowledge. It's not just learning the knowledge-it's actually acquiring the knowledge. We try to do this in our own environment. We wanted to move away from the dump truck of knowledge and by doing so, and engage the learners in accountability and preparation. We call this flipping the classroom, whereby the learner is responsible to come prepared, to ask questions, to be engaged. It allows the faculty, who are traditionally the transmitters of knowledge, to become coaches.

Over 12 educational sessions, a resident-faculty dyad was created. They were responsible for creating the content of conference and leading the discussion. Case-based discussions were used similar to the ones you've seen and derived from the thoracic surgery curriculum cases. The readings were expected to be done before the conference and the cases that were going to be used were predistributed for preview to the residents and faculty. This is a brief video, and I don't want you to focus on the exact content of this conference but to understand that it involved a wide range of content that was accessible to learners of all levels so we could engage our medical students, our junior residents, our fellows, etc, to participate and remain engaged. As you can see, as this conference goes on, the complexity of concepts continues to increase such that we can use a wide range of teaching principles. At the end of every conference, a quiz was prepared by the faculty member who was supervising. It was based on the curricular readings and not limited to just what was in the conference itself.

All residents in our program were required to complete the quiz and controls were created by using the faculty, the thoracic, or cardiac faculty, to compare the resident scores too. Each quiz also had a brief survey, which talked about their preparation preview, attendance, and self-assessment. The quiz results were then reported back to the residents and to the faculty. Here's an example quiz. The quizzes were of various different formats, different types of questions, not designed for some of the feedback but, in fact, formative feedback. At the conclusion of this, we distributed a survey, which included resident demographics, career choices, etc. Analysis of accuracy of the quizzes was assessed by linear regression with adjustment for clustering by responses of residents and faculty. Interactions between time and each of faculty and seniority were evaluated for scores and predicted test scores were created to summarize the average effects. 
We have a total of 10 residents in our program, 4 in their last 2 years of training and 6 with more than 2 years of training, and we arbitrarily divided them along those lines. Most were male, and their career interests were relatively evenly distributed across our subspecialties. A total of $50 \%$ reported an academic career interest. For the control group, there were 6 cardiac faculty completing the quizzes and 5 thoracic faculty completing the quizzes. In terms of preparation and engagement, as you can see, most residents were able to read a good portion of the curricula material before attending the conference. The majority of residents were able to preview the quizzes at some level before attending the conferences. As is the case in many of our weeks, there were a number of residents that were off site or off service and were not able to attend our conference but more than half either attended or presented. There was a reasonable distribution of resident self-assessment of competence in terms of how they thought they were in that particular topic. Not surprisingly, using linear regression, we found that reading from the curriculum resulted in greater quiz scores, reviewing the quiz resulted in greater quiz scores, and selfassessment, increasing self-assessment resulted in greater quiz scores.

Attendants of conference did not suggest that preparation was very important, but we also felt that this was a trend that would have seemed to be significant with greater numbers. We fit test scores using a model and found that each element of engagement and preparation resulted in about a $5 \%$ increase in quiz scores, not entirely surprising, but supported by the data. As you can see, as I said, about 5\% per topic. Individual quiz scores across the weeks were compared by resident and faculty. In some weeks, residents outscored the faculty and vice versa. When we compared them, there was no statistical difference between residents and faculty over the course of the entire assessment. However, the residents did improve with time, suggesting that they learned how to use the system to their advantage whereas faculty scores remained about level. Somewhat surprisingly, junior residents outperform the senior residents across all levels, again, suggesting that they had better preparation coming into conference likely due to work hour restrictions and availability. However, both groups of residents improved at about the same rate.

We attempted a program director's report, and this was one of the hardest things to do in this experiment. This is the report you see in the abstract book. We actually abandoned this fairly quickly because it was not actually a useful tool for us to interpret. Residents did give us feedback. The majority viewed this as superior. The majority viewed this as a motivator. All of them sought feedback and reported increased faculty engagement. The feedback was criticized as being not timely enough and not enough discussion about correct answers. The program director's report needs improvement and this took a lot of time. The limitations are that this is a single study, a single-center study. There was no quiz validation. Sustainability was not studied and we can't translate this into competence or pass rates. This obviously took a lot of time. A new learning management system is launching on July 1, which will allow for benchmark quizzes, usage statistics, and direct feedback, and more cases are being added regularly. We look forward to a multicenter study.

Adult learning theory requires preparation, engagement, and participation for the learners and can be achieved at a local level. All residents improved over time. Faculty was more engaged. The new TSC platform may allow for improved multicenter data analysis to support these findings.

I'd like to thank the Association for the opportunity to present our work.

Moderator: Discussion will be opened by Dr Craig Baker.

Dr Baker. Thank you. I want to congratulate Dr Mokadam and his co-authors from the University of Washington for an excellent and stimulating presentation today. I want to thank them for allowing me to review the manuscript well in advance and I really want to thank Nahush on behalf of everyone involved in thoracic surgery education for his substantial contributions to our specialty and for elevating the excitement and importance of thoracic surgery education, not just nationally but internationally as well. The thoracic surgery curriculum was never meant to be prescriptive but to be used to allow programs to structure a curriculum that could be successfully implemented vocally as you have done.

Nahush, as you have eloquently pointed out, the flip classroom is an educational strategy and essentially reverses traditional learning by delivering content outside of the classroom and engages the learner in the classroom with the guidance of a mentor. Although this strategy may seem intuitively beneficial to some educators, in reality, this concept has origins only dating back to 1993 and was popularized ultimately by the Kahn academy, which essentially has become synonymous with the flip classroom strategy. Therefore, I have 3 questions, which I will ask you separately.

First, you are at an institution with substantial educational infrastructure and the senior faculty member sitting next to me who has worked tirelessly during the last decade to transform thoracic surgery education 
in this country. Undoubtedly, your ability to institute the flip classroom model is predicated on the development of your extensive library of case presentations, which were developed or at least fine-tuned locally. Although those presentations that you say have been made available nationally, they haven't really been used widely, and some critics argue that using someone else's PowerPoint lecture that they didn't prepare is difficult. How, therefore, do you envision making this potentially superior educational strategy more widely adopted understanding that most institutions may not have the infrastructure available to implement it?

Dr Mokadam. Thank you for your comments, Craig, and I always appreciate your support. I agree with you. This was a very time-intensive strategy. With the cases that are available now, because they're coming down as PowerPoint slides, they're not coming down as PDFs, they are modifiable. They can serve as a platform for anybody to use to adapt to their own needs to use as a launching point for discussion. I appreciate and understand the criticism, but I have downloaded somebody else's talk not done at our institution and modified it to my purposes for teaching. It's not hard to do. It does take a little bit of work but it's not hard to do and can be done if we think it's important.

Dr Baker. Yeah, I agree with you. I think a lot of this is getting the message out and just showing people that these are easy to use and these people have spent countless hours so they don't have to reinvent that wheel. Second, it is notable that the Kahn academy specifically uses online videos as the learning medium similar to the initial efforts of the TSDA dating back to the early 2000s. The joint council subsequently developed a more robust curriculum with book chapters, similar manuscripts, online videos, and lectures. You point out in your manuscript that the majority of residents completed the reading, but completing the reading, as you and I know, and truly mastering that knowledge in them are quite different. The idea of the flip "mastery" classroom takes the concept of the flip classroom one step further requiring each student to master a topic before progressing to the next assignment. Do you foresee, as we're about to launch the new learning management system, that you will or we should mandate our learners demonstrate some type of mastery of this knowledge before them being permitted to progress or at least, let's say, come to your structured conference?

Dr Mokadam. That's a great question and point and it's, I think, the subject of what we call competency. How do we measure competency and do with demand competency before advancement? There's obviously a lot of controversy in that we currently have a time-based completion of our curriculum, a 2-year program, a 3-year program, or an integrated 6-year program, that's not subject to competency based advancement unless it's really egregious. Are we willing, as a community, to do that? I think that's a bigger question for people with bigger hats than mine. Currently, we demand adequate competency. We use our milestones. Have you achieved level 1, 2, 3, 4, 5? How are we going to do that in a broader population, I think, is harder to answer in this room.

Dr Baker. Yeah, I think we're not going to tell our residents they can't come to conference because they didn't master something but I do think what you've done is remarkable and I do think we can build in some type of free conference proof that they've really tried to understand the materials so that when they get there, it's different. My last question is a little simpler. You mentioned that the majority of residents viewed this message favorably. Obviously that means that some didn't. Can you elaborate on the criticisms of those residents that actually didn't like all the efforts you put into doing this?

Dr Mokadam. Yeah, we struggled with that for a long time and I didn't find the answer out to that until our annual program review when we had a couple of residents sitting in the room and we asked them directly, you know, what do you think about conference. It was actually the time, the amount of time that the residents put into preparing these conferences even with the faculty member can be overwhelming. They're all highly motivated residents. Some of them are sitting in this room and they want to do a good job. They want to come to conference prepared and they have all of their clinical activities so the preparing resident actually really feels the heat of getting it done and I think that was the cause of some of them saying, well, you know, that's a lot of work. Maybe it would be really nice to have an outside lecture so I don't have to do that this week.

Dr Baker. Well, again, great job and take it one step further. Thanks, Nahush.

Dr Mokadam. Thank you.

Moderator: David?

Dr Fullerton. Hi. This was a very elegant education improvement study that you all performed. I guess I have 2 questions. One is a comment. You know, the most viable commodity in graduate medical education is time. It's more valuable than money. When you look at the 80 -hour work week, 80-hour work week is a lot of time. Then, even when you go home, a resident goes home, they need time for their brain to unwind and to recover from a difficult residency. Although the majority of the residents completed the reading before the conferences, $25 \%$ either did little reading or not at all. Therefore, $25 \%$ of the residents came unprepared for this. Then, the second number is, I believe, $35 \%$ didn't attend the conferences so they were off somewhere or maybe on vacation, I don't know. My question is, do you provide carve outs for the residents in regards to doing the reading, protected time almost, to do the reading, and prepare for these conferences and also to attend the conferences? Are the conferences mandatory or are they highly encouraged? 
Dr Mokadam. It's a little bit of both. You're right. Some of the residents did not come to conference. Remember, in an integrated program, a lot of our rotations are not on cardiothoracic surgery, especially at the junior level. The dropouts of people not being there, I think, were largely the junior residents. The survey was anonymous so I can't answer that directly. That being said, we we're working with our general surgery program to increase resident attendance to our conferences in the junior levels. You're absolutely right about the other point about conference preparation, reading. The way the residents responded though in the survey was that this was a motivator, so I suspect if we hadn't done this, preparation would have been even less although $25 \%$ lack of preparation sounds bad, I think it's probably a lot better than it would have been had we not done this.

Moderator: Dr Whyte?

Dr Whyte. Thanks very much. Nahush, you did a great job and a great presentation. I want to pile on to David a little bit, what he's saying. I mean, we run a similar sort of conference. We have faculty do case presentations. We have expectations that the residents do the curricular reading ahead of time. My experience is similar to yours in that you pointed out that a third of residents, only a third did most or all of the reading. Now, when I do one of those conferences, I do all the reading and maybe I can do it faster because I've got a better baseline knowledge but I don't know how to motivate them to do all of that reading. I think there's a week or so between conferences. It's not that much, but the one you showed was a lot of book chapters but the reality is when you look at those, there's a fair amount of overlap between chapters on many occasions. I struggle to get them to motivate them to do all the readings. I don't think it's that hard to do and I continually am impressed by when they come to conference and they're not prepared and we ask them questions in a Socratic method. It doesn't really faze them that they don't know the answer. I mean, I think when I was a resident, I'd be mortified if I didn't know the answers to some of these questions. It's like oh, you know, I don't know.

Dr Mokadam. Well, I think that's ...

Dr Whyte. That's a great ... How do you do that? How do you do that and how can we do that better?

Dr Mokadam. I think that's where the quizzes really come in handy because if they know they're getting tested on it and they're going to get feedback on it, and their program director is going to see the results of that quiz, that's a really good motivator. Our residents actually really glommed onto the quizzes. The type of feedback they saw after completing the quizzes like I want to know what I got, I want to know what I got. What was my answer? What did I get wrong? I want to know the right answer. That was actually a really good motivator for them. I think that with the new curriculum coming out, the ability to do benchmark quizzes is going to help us motivate our residents to prepare better and say okay, look, on Friday, you're getting a benchmark quiz. We're going to see the result and we're going to compare you across everybody else in the country.

Moderator: We're going to have to stop now. Thank you very much.

Dr Mokadam. Thank you. 
APPENDIX E1. SURVEY ITEMS

Survey Items for Flipping the Classroom: CaseBased Learning, Accountability, Assessment, and Feedback Leads to a Favorable Change in Culture

1. Year of training:

2. Sex:

3. Last (thoracic) American Board of Surgery In-Training Exam score:

4. Career interest (specialty):

5. Career interest (academic vs private):

6. Residency format (I6, traditional, 4/3):

7. Which conference format is most educationally valuable?

(Please rank 1-6, 1 being most valuable and 6 being least valuable.)

Resident didactic presentation (ppt)

Faculty didactic presentation (ppt)

Case-based format

Self-Education Self-Assessment in Thoracic Surgery

Guest lecturer from outside the division

Other

8. Does the case-based format change your preparation for conference (yes, no)?

9. Do you find the reading assignments for the weekly thoracic surgery curriculum 88 curriculum (too much, too little, just right)?
10. Do you find the readings assigned for the weekly University of Washington case-based conferences (too much, too little, just right)?

11. Do you preview the distributed cases prior to conference (no, less than $50 \%$, more than $50 \%$ )?

12. Have your self-study habits changed over the course of this pilot project (no, less preparation, more preparation, more self-study)?

13. Have you reviewed the cases after conference with their content (no, less than 50\%, more than 50\%)?

14. Do the quizzes adequately reflect the reading or case presentations (yes, no)?

15. Do the quizzes encourage you to prepare for conference (yes, no)?

16. The difficulty level of the quizzes is (too easy, just right, too hard)?

17. What are barriers to completing the quizzes (clinical duties, sleep deprivation, not educational, performance anxiety, other)?

18. When do you typically complete the quizzes (immediately after conference, immediately after review, whenever there is time)?

19. What aspects of reporting would be most valuable to your learning (write in)?

20. How should we improve this process (write in)?

21. How do you plan to change your learning habits in response to this (write in)?

22. How can the faculty improve their interaction with you to optimize your conference learning (write in)? 